\title{
Rare Huge Congenital Intracranial Silent Teratoma in Older People: A Case Report
}

\author{
Yeting Zhou ${ }^{1}$, Daoming Tong ${ }^{2}$, Xiaodong Chen ${ }^{2}$, Guangsheng Wang ${ }^{2}$, Tonghui Yang ${ }^{2}$, Yuanwei Wang ${ }^{2}$ \\ ${ }^{1}$ Medical Evaluation Unit, Shuyang People'Hospital, Shuyang, China \\ ${ }^{2}$ Departments of Neurology, Shuyang People’Hospital, Shuyang, China \\ Email: tongdaoming@163.com
}

Received August 2, 2013; revised September 2, 2013; accepted September 10, 2013

Copyright (C) 2013 Yeting Zhou et al. This is an open access article distributed under the Creative Commons Attribution License, which permits unrestricted use, distribution, and reproduction in any medium, provided the original work is properly cited.

\begin{abstract}
The huge congenital intracranial teratoma is very rare. We report a case of a 77 years old male with giant congenital brain teratoma without clinical symptoms. His computed tomography (CT) scan showed a massive tumor $(6 \times 5 \times 6 \mathrm{~cm})$ of irregular high density (inside with some point flaky low density) in left temporal lobe region, the lower corner of the left ventricle downward shift, and enhanced CT scan no enhancement. Magnetic resonance imaging (MRI) on brain showed an irregular huge tumor in the left temporal lobe area, with multiple nodular or lobulated mixd high and low or equal signal changes, in which the liquid signal based. The midline was shift to the right, and the posterior horn of the left ventricle was compressed and downward shift, expansion, and hydrocephalus. After follow-up 12 months, the patient presents a normal daily life and work and no neurological signs as usual. Our observations show that the huge tumor in brain with multiple nodular or lobulated variety of mixed signal changes on MRI without symptoms is a congenital intracranial silent teratoma.
\end{abstract}

Keywords: Tumor; Congenital Intracranial Teratoma; Computed Tomography; Magnetic Resonance Imaging; Outcome

\section{Introduction}

Huge congenital intracranial teratoma is a rare intracranial benign tumor and often occurs in the prenatal period $[1,2]$. Computed tomography (CT) and magnetic resonance imaging (MRI) are useful for detecting the localization and delineating the extent of tumor. Although several studies reported that CT and MRI were a sensitive and specific tools for diagnosing congenital intracranial teratoma in newborn, infant, or stillborn [1-3], there has been very little discussion about the details of the radiological features of huge congenital intracranial teratoma in older people. We report the case of an old age patient who was found to have a rare huge congenital teratoma arising in the intracranial brain with brain shift without symptoms, and the present imaging findings are obtained by CT and MRI.

\section{Case Report}

A 77-year-old male was admitted to our department of neurology in August 8, 2012, due to a two months history of recurrent headaches. He had a 20-year history of chronic bronchitis and six months of a hypertension, but no any history of headaches or seizures. There was no vomiting or daily life limited. The blood pressure was $170 / 100 \mathrm{mmHg}$, the pulse 80 , and the respiratory 22 . His Consciousness and intelligent was normal, and his other neurological (such as the size and reactive to light of pupils) and medical examination was also no abnormality. The laboratory examination results including liver, kidney function, and electrolytes were normal. The blood glucose was $3.97 \mathrm{mmol} / \mathrm{l}$, with normal blood lipids. His ECG showed myocardial ischemic changes.

On non-contrast enhanced cranial CT scan, the giant massive of irregular high density $(6 \times 5 \times 6 \mathrm{~cm})$ was found in left temporal lobe region where there was some point flaky low density, and the posterior horn of the left lateral ventricle downward shift (Figure 1A); enhanced CT scan no enhancement. Magnetic resonance imaging (MRI) on brain showed an irregular huge tumor in the left temporal lobe area, the midline shift to the right, the posterior horn of the left ventricle compressed downward shift, expansion, and hydrocephalus (Figure 1B). The huge tumor presented extending to the infratentorial 
brainstem, and obviously midbrain compressed and displacement, and multiple nodular or lobulated mixd high and low or equal signal, in which the liquid signal based (Figure 1C). Enhanced MRI was without enhancement. The huge congenital intracranial teratoma was diagnosed. On follow-up a year, in addition to occasional headache, He was no other abnormalities and as usual in the daily life and work.

\section{Discussion}

Our patient was an over 70-year-old of patient who underwent brain imaging study due to recurrent headache in recent months. The imaging results are surprising to find that there is a huge tumor in the left temporal lobe area, with significant the brain and upper brainstem compression and displacement which resembles herniation. The patient did not be daily activities limited, no vomiting, and no disturbance of consciousness and other neurological signs. In addition, the patient was no previous history of cerebral disease, and he had been able to engage in normal daily work and life. Therefore, the diagnosis of herniation and increased intracranial pressure can not be considered. In the literature, congenital intracranial teratoma is a rare benign tumor, often in the prenatal period have occurred [1,2]. The teratoma may lead to loss of brain compensatory function, and emergence the neurological symptoms of localized compression or obstruction if rapidly growth. However, we believe that present this huge intracranial tumor is born in the brain of patients, and long-term potential congenital benign tumors without any symptoms due to slowly growth.

Previous imaging studies have shown that brain teratoma is characterized by multiloculated or cystic mass or

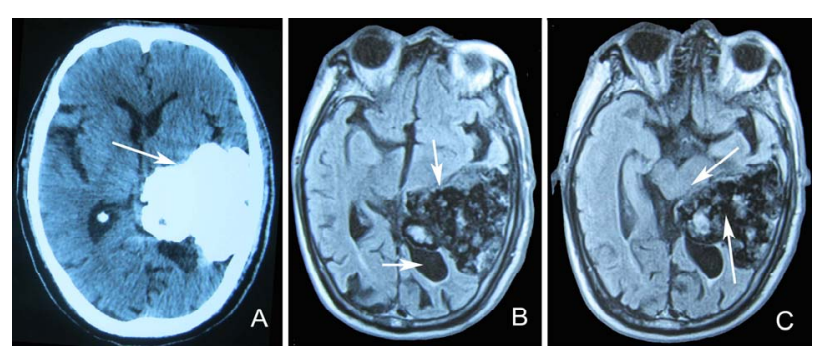

Figure 1. A 77 years old patient with congenital brain teratoma brain imaging. Non-contrast enhanced CT scans show a $6 \times 5 \times 6 \mathrm{~cm}$ huge tumor of irregular high density in the left temporal lobe of the patient (A). MRI show an irregular huge tumor in left temporal lobe area (B. upper arrow), with the midline shift to the right, the posterior horn of the left ventricle compressed downward shift, expansion, and hydrocephalus (B. below arrow), and extending to the infratentorial brainstem, resulting in midbrain compression and displacement (C. upper arrow); on FLAIR show multiple nodular or lobulated mixed high and low or equal signal, in which the liquid signal based (C. below arrow). a heterogeneous composition changes, including liquid ingredients, fat, soft tissue, or calcification [3], and distinguishing the interface between the tumor and cartilage, bone, brain, and fluids [4]. Our patient's imaging is consistent with the imaging features of brain teratoma, so the diagnosis of intracranial teratoma is established.

According to our patient's MRI findings, the brain shift was sufficient to reach a degree of herniation. However, the patient had no neurological symptoms. One likely explanation is that the growth of brain teratoma with developmental brain may be increased over time, whereas its growth over time may be also stopped when the developmental brain is stopped. It is not only during the developmental brain without clinical symptoms due to a slowly compensatory and adaptation of brain functions, but also is no symptoms during the long-time of developmental brain stopped.

After Follow-up 12 months, the patient presents the normal activities and no neurological signs, which further confirms that this huge brain teratoma is a benign or silent. Because of the patient with a history of hypertension, perhaps his recurrent headache is associated with the reversible cerebral vasoconstriction syndrome [5] or transient ischemic attack (TIA) [6].

Brain teratoma is a congenital ectopic tumor with brain growth synchronization, which is a derivative from the head end of embryonic neural tube. Giant congenital intracranial teratoma is rare, however, asymptomatic huge congenital brain teratoma in older people has not been reported. Our findings confirm that the outcome of huge congenital intracranial teratoma is good, and there is no need for surgery if without neurological symptoms.

\section{REFERENCES}

[1] Y. H. Chien, P. N. Tsao, P. N. Lee, S. F. Peng and K. I. Yau, "Congenital Intracranial Teratoma," Pediatric Neurology, Vol. 22, No. 1, 2000, pp. 72-74. http://dx.doi.org/10.1016/S0887-8994(99)00103-4

[2] F. A. Soares, S. E. Fischer, M. A. Reis and E. G. Soares, "Massive Intracranial Immature Teratoma. Report of a Case with Polyhidramnios and Intense Pelvic Pain," Arq Neuropsiquiatr, Vol. 54, No. 2, 1996, pp. 309-312.

[3] N. Isik, S. Yildirim, M. Onoz and A. Aras, "Surgical Treatment of Huge Congenital Extracranial Immature Teratoma: A Case Report," Child's Nervous System, Vol. 27, No. 5, 2011, pp. 833-839.

http://dx.doi.org/10.1007/s00381-010-1335-3

[4] G. Hedlund, "Congenital Frontonasal Masses: Developmental Anatomy, Malformations, and MR Imaging,” Pediatric Radiology, Vol. 36, No. 7, 2006, pp. 726-727. http://dx.doi.org/10.1007/s00247-005-0100-3

[5] Headache Classification Committee of the International Headache Society (IHS), "The International Classification of Headache Disorders,” 3rd Edition, Cephalalgia, Vol. 
33, No. 9, 2013, pp. 629-808.

[6] P. Kropp, M. Holzhausen, E. Kolodny, et al., "Headache as a Symptom at Stroke Onset in 4431 Young Ischaemic Stroke Patients. Results from the Stroke in Young Fabry
Patients (SIFAP1) Study," Journal of Neural Transmission, 2013. http://dx.doi.org/10.1007/s00702-013-1014-0 